\title{
SPECTRAL INDICES AND PARTICLE AGEING STUDIES ON THE GIANT RADIO GALAXY NGC6251
}

\author{
K.-H. MACK ${ }^{1}$, U. KLEIN ${ }^{1}$, L. SARIPALLI ${ }^{1,2}$, A. G. WILLIS ${ }^{3}$ \\ AND C. P. O'DEA ${ }^{4}$ \\ 1 Radioastronomisches Institut der Universität Bonn - Auf \\ dem Hügel 71, 53121 Bonn, Germany \\ 2 Indian Institute of Astrophysics - Koramangala, Bangalore \\ 560034, India \\ 3 Dominion Radio Astrophysical Observatory - P.O. Box 248, \\ Penticton, BC, Canada V2A $6 \mathrm{~K} 3$ \\ 4 Space Telescope Science Institute - 3700 San Martin Drive, \\ Baltimore, MD 21218, USA
}

\section{Introduction}

In the framework of our high-frequency survey of giant radio galaxies with the Effelsberg 100-m telescope (Klein et al., 1994; Saripalli et al., 1995) we have obtained radio continuum maps of NGC6251, a source of $1.5 \mathrm{Mpc}$ size $\left(\mathrm{H}_{0}=75 \mathrm{~km} \mathrm{~s}^{-1} \mathrm{Mpc}^{-1}\right)$. Together with low-frequency WSRT observations (Willis \& O'Dea, 1990), these measurements form a unique data base which for the first time allows thorough studies of the spectral index over a large frequency range. Theoretical models of particle ageing have been fitted to the spectrum to determine particle ages and other relevant physical parameters. Because of the immense size of NGC6251 these numbers provide information about the physics of the surrounding intergalactic medium.

\section{Spectral index distribution and particle ageing}

At low frequencies, steep spectra are found in the outer lobe regions with mean values of $1.4\left(S_{\nu} \sim \nu^{-\alpha}\right)$ in the south-eastern lobe. Significant spectral asymmetries could not be found despite the asymmetric morphology and in contrary to other sources of this species. The core has a typical flat spectrum $(\alpha=0.3)$. The jet spectrum is very characteristic $\left(\alpha_{\text {mean }}=\right.$ 0.6 ). Along the jet a gradual steepening is seen up to $\alpha=0.8$ where it 
widens and mixes with older lobe particles. Both hot spot regions possess spectra of $\alpha=0.55$, close to the theoretical values expected for shockaccelerated particles. The high-frequency maps qualitatively show a similar behaviour, with a slight indication of increasing spectral indices. Similar to the work of Carilli et al. (1991) we have tried to fit a model spectrum to all data points in order to derive the break frequency $\nu_{B}$ across the source. The break frequency is used to evaluate the particle ages at various positions over the source, assuming that the dominant energy losses are due to synchrotron and Inverse Compton processes, neglecting adiabatic expansion and particle reacceleration. We have applied the model by Jaffe \& Perola (1973) which allows for permanent pitch angle isotropization. The lowest break frequency which could be derived from three frequencies lies around $3 \mathrm{GHz}$ and is found at the southern edge of the north-western lobe. Since at lower frequencies the emission is much more extended and could not be detected at high frequencies, we have to take this value as an upper limit. Using a minimum energy magnetic field of $0.6 \mu \mathrm{G}$ for the lobes and a field equivalent to the microwave background of $3.4 \mu \mathrm{G}$ we obtain a minimum source age of $\tau=6 \cdot 10^{7} \mathrm{yrs}$.

\section{NGC6251 as probe for the intergalactic medium}

Because of the immense size of NGC6251 it can serve as a probe of the surrounding intergalactic medium (IGM). Following the example of Lacy et al. (1993) we have estimated the density of the IGM by balancing the momentum flux of the jet against the ram pressure of the external medium. The most uncertain parameter is the size of the effective area, the piston impinging on the IGM. We identify the effective area with the hot spot area, whose radius is assumed to be $2.5 \mathrm{kpc}$, which is an upper limit. The source advance speed was determined by dividing the source radius by the source age yielding a mean velocity of $0.045 \mathrm{c}$. Using these parameters we obtain a particle density $n_{e} \sim 6.2 \cdot 10^{-6} \mathrm{~cm}^{-3}$ for the ambient medium. This value is lower than what is generally inferred for GRGs, but may be in accord with the fact that the jet of NGC6251 is obviously subject to strong influences by the surrounding medium, as evidenced by the various distortions and changes in brightness.

\section{References}

Carilli C. L., Perley R. A., Dreher J. H., and Leahy J. P. (1991) ApJ, 283, 554

Jaffe W. J. and Perola G. C. (1973) $A \mathcal{B} A, 26,423$

Klein U., Mack K.-H., Strom R., Wielebinski R., and Achatz U. (1994) $A \& A, 283,729$

Lacy M., Rawlings S., Saunders R., and Warner P. J. (1993) MNRAS, 264, 721

Saripalli L., Mack K.-H., Klein U., Strom R., Singal A. K. (1995) $A \& A$, in press

Willis A. G. and O'Dea C. P. (1990) IAU Symp., 140, 455 